\title{
Copulatory courtship song in Lutzomyia migonei (Diptera: Psychodidae)
}

\author{
Felipe M Vigoder ${ }^{1}$, Nataly A Souza ${ }^{2}$, Alexandre A Peixoto ${ }^{1,3} /+$ \\ 'Laboratório de Biologia Molecular de Insetos ²Laboratório de Transmissores de Leishmanioses, Instituto Oswaldo Cruz-Fiocruz, \\ Av. Brasil 4365, 21045-900 Rio de Janeiro, RJ, Brasil IInstituto Nacional de Ciência e Tecnologia em Entomologia Molecular, Brasil
}

Lutzomyia migonei is a vector of leishmaniasis with a wide distribution in South America, which could favour population differentiation and speciation. Cryptic species of the Lutzomyia longipalpis complex, the widely distributed sand fly vector of visceral leishmaniasis in Latin America, have previously been shown to display distinct copulation songs. We found that Lu. migonei males also produce a song during copulation. This "lovesong” presents short trains (6-8 pulses) with an inter-pulse interval around $26 \mathrm{~ms}$ and is potentially involved in cryptic female choice and insemination success.

Key words: sand flies - lovesong - leishmaniasis

Lutzomyia migonei is a vector of American cutaneous leishmaniasis (Rangel \& Lainson 2009) and it was recently suggested that $L u$. migonei may also be a vector of American visceral leishmaniasis (de Carvalho et al. 2010, Salomon et al. 2010). This sand fly has a wide distribution in South America, being found from Argentina to Colombia across a variety of habitats (Rangel \& Lainson 2003, 2009). The extensive distribution of this species may contribute to population differentiation and speciation due to geographical isolation and local adaptation, especially when in association with sexual selection (Ritchie 2007).

Lutzomyia longipalpis, a sand fly species that is also distributed over a large area in South and Central America, is a complex of sibling species (Ward et al. 1988, Lanzaro et al. 1993, Bauzer et al. 2002, 2007, Arrivillaga et al. 2003, Maingon et al. 2003, 2008). One important marker that has been used to differentiate the cryptic sibling species of this complex occurring in Brazil is the song produced by males during copulation (Souza et al. 2004, Araki et al. 2009). Differences in this copulatory courtship song probably contribute to the insemination failure observed in crosses between some Brazilian populations of this vector (Ward et al. 1983, 1988, Souza et al. 2008). Indeed, acoustic signals in insects are often involved in sexual selection and reproductive isolation of closely related species (Ewing 1989).

Copulation songs were also found in males of Lutzomyia cruzi (Vigoder et al. 2010) however, this species is very closely related to Lu. longipalpis s.l. and should

Financial support: Howard Hughes Medical Institute, CNPq, FAPERJ, CAPES, FIOCRUZ

+ Corresponding author: apeixoto@ioc.fiocruz.br

Received 11 August 2010

Accepted 10 November 2010 be considered a member of this complex (Vigoder et al. 2010). To the best of our knowledge, copulation songs have not been previously reported in other sand fly species. In the present paper we report that $L u$. migonei males also produce a song during copulation.

Recordings were carried out using a Sony Hi8CCD-TRV65 video camera and Sony SLV-77HFBR VCR, according to the procedure outlined by Souza et al. (2004). Briefly, a male and female virgin were placed inside the INSECTVOX (Gorczyca \& Hall 1987) at $25^{\circ} \mathrm{C} \pm 1{ }^{\circ} \mathrm{C}$ for about $5 \mathrm{~min}$. If no copulation occurred during this time, the couple was replaced for another one. Insects used in the present work were the $\mathrm{F} 1$ of females collected in Mesquita, state Rio de Janeiro, Brazil (22 $\left.{ }^{\circ} 46^{\prime} \mathrm{S} 43^{\circ} 25^{\prime} \mathrm{W}\right)$, an area of cutaneous leishmaniasis transmission (Meneses et al. 2002) and identified according to Young and Duncan (1994). A CED1401 A/D converter was used to digitalize the acoustic signals that were analyzed using Spike2 software (v4.08), both from Cambridge Electronic Design (UK). Five parameters were analyzed from the song of six different males: inter-pulse interval (IPI) and train length (TL), measured in milliseconds (ms), number of pulses per train (NP) and carrier frequency (FREQ), in hertz (Hz), and number of cycles per pulse (CPP).

We observed that $\mathrm{Lu}$. migonei males also sing during copulation, similar to Lu. longipalpis s.l. (Souza et al. 2004, Araki et al. 2009). Figure 1A shows a segment of $2.5 \mathrm{~s}$ of the song of a Lu. migonei male with four trains and Figure 1B shows a single train in more detail. $L u$. migonei males produce several short TL $(153.44 \pm 7.92$ $\mathrm{ms})$ composed of a small number of NP $(6.95 \pm 0.18)$ with a short IPI $(25.87 \pm 0.83 \mathrm{~ms})$. These three song parameters make the copulation song pattern of Lu. migonei quite different from those observed in Lu. longipalpis s.l. (Souza et al. 2004, Araki et al. 2009). The song of $L u$. migonei has also a low FREQ $(280.87 \pm 15.62 \mathrm{~Hz})$ and has pulses about three CPPs ( $3.28 \pm 0.12)$.

The Lu. migonei male song produced during copulation is probably part of courtship and potentially in- 
A

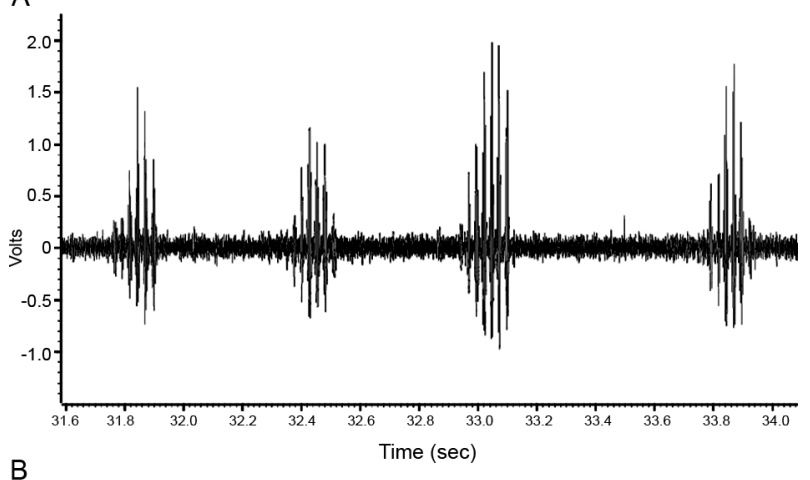

B

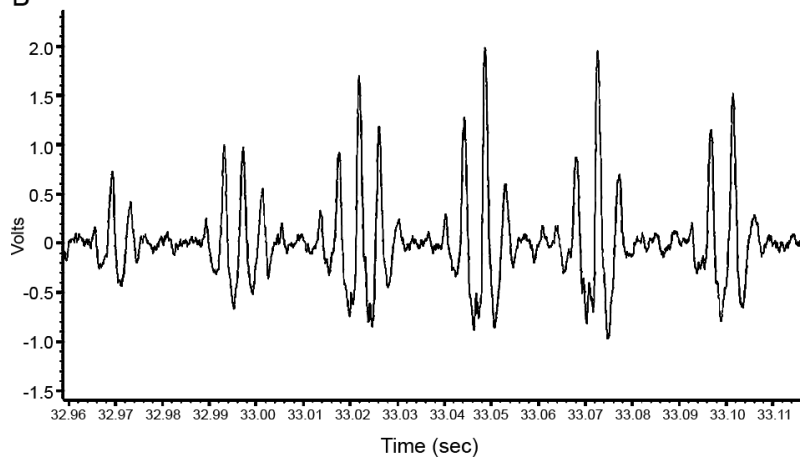

Lutzomyia migonei copulatory courtship song. A: segment of $2.5 \mathrm{sec}$ of song with four trains; B: single train in more detail.

volved in cryptic female choice and reproductive isolation (Eberhard 1996). This has been shown in other insect species whose males also sing during copulation, such as Drosophila birchii and Drosophila serrata (Hoikkala \& Crossley 2000, Hoikkala et al. 2000).

$L u$. migonei and $L u$. longipalpis are not very closely related (Mazzoni et al. 2002, Beati et al. 2004) and in fact some authors place the former species in a new genus, Migonemyia, proposed by Galati (1995). This suggests that copulatory courtship songs might be widespread in sand flies and could prove to be a powerful tool in studies aiming at disclosing cryptic species. In this respect, it would be interesting to record the song of males from populations of $\mathrm{Lu}$. migonei from different geographical locations, including the areas where this species might be transmitting visceral leishmaniasis (Salomon et al. 2010), to see if there are differences that might suggest that this important vector is in fact a complex of cryptic species.

\section{REFERENCES}

Araki AS, Vigoder FM, Bauzer LG, Ferreira GE, Souza NA, Araújo IB, Hamilton JG, Brazil RP, Peixoto AA 2009. Molecular and behavioral differentiation among Brazilian populations of Lutzomyia longipalpis (Diptera: Psychodidae: Phlebotominae). PLoS Negl Trop Dis 3: e365.

Arrivillaga J, Mutebi JP, Pinango H, Norris D, Alexander B, Feliciangeli MD, Lanzaro GC 2003. The taxonomic status of genetically divergent populations of Lutzomyia longipalpis (Diptera: Psychodidae) based on the distribution of mitochondrial and isozyme variation. $J$ Med Entomol 40: 615-627.
Bauzer LG, Gesto JS, Souza NA, Ward RD, Hamilton JG, Kyriacou CP, Peixoto AA 2002. Molecular divergence in the period gene between two putative sympatric species of the Lutzomyia longipalpis complex. Mol Biol Evol 19: 1624-1627.

Bauzer LGSR, Souza NA, Maingon RDC, Peixoto AA 2007. Lutzomyia longipalpis in Brazil: a complex or a single species? A mini-review. Mem Inst Oswaldo Cruz 102: 1-12.

Beati L, Cáceres AG, Lee JA, Munstermann LE 2004. Systematic relationships among Lutzomyia sand flies (Diptera: Psychodidae) of Peru and Colombia based on the analysis of $12 \mathrm{~S}$ and 28S ribosomal DNA sequences. Int J Parasitol 34: 225-234.

de Carvalho MR, Valença HF, da Silva FJ, de Pita-Pereira D, de Araújo Pereira T, Britto C, Brazil RP, Filho SP 2010. Natural Leishmania infantum infection in Migonemyia migonei (França, 1920) (Diptera: Psychodidae: Phlebotominae) the putative vector of visceral leishmaniasis in Pernambuco State, Brazil. Acta Trop 116: 108-110.

Eberhard WG 1996. Female control: sexual selection by cryptic female choice, Princeton University Press, New Jersey, 472 pp.

Ewing AW 1989. Arthropod bioacoustics, Cornell University Press, New York, 260 pp.

Galati EAB 1995. Phylogenetic systematics of the Phlebotominae (Diptera, Psychodidae) with emphasis on American groups. Bol Dir Malariol Saneam Amb 35: 133-142.

Gorczyca M, Hall JC 1987. The INSECTAVOX, an integrated device for recording and amplifying courtship songs of Drosophila. Dros Inf Serv 66: 157-160.

Hoikkala A, Crossley S 2000. Copulatory courtship in Drosophila: behavior and songs of Drosophila birchii and Drosophila serrata. J Insect Behav 13: 71-86.

Hoikkala A, Crossley S, Castillo-Melendez C 2000. Copulatory courtship in Drosophila birchii and D. serrata, species recognition and sexual selection. J Insect Behav 13: 361-373.

Lanzaro GC, Ostrovska K, Herrero MV, Lawyer PG, Warburg A 1993. Lutzomyia longipalpis is a species complex: genetic divergence and interspecific hybrid sterility among three populations. Am J Trop Med Hyg 48: 839-847.

Maingon RD, Ward RD, Hamilton JG, Bauzer LG, Peixoto AA 2008. The Lutzomyia longipalpis species complex: does population sub-structure matter to Leishmania transmission? Trends in Parasitol 24: 12-17.

Maingon RDC, Ward RD, Hamilton JGC, Noyes HA, Souza N, Kemp SJ, Watts PC 2003. Genetic identification of two sibling species of Lutzomyia longipalpis (Diptera: Psychodidae) that produce distinct male sex pheromones in Sobral, Ceará State, Brazil. Mol Ecol 12: 1879-1894.

Mazzoni CJ, Gomes CA, Souza NA, de Queiroz RG, Justiniano SC, Ward RD, Kyriacou CP, Peixoto AA 2002. Molecular evolution of the period gene in sandflies. J Mol Evol 55: 553-562.

Meneses CR, de Azevedo AC, da Costa SM, Costa WA, Rangel EF 2002. Ecology of American cutaneous leishmaniasis in the state of Rio de Janeiro, Brazil. J Vector Ecol 27: 207-214.

Rangel EF, Lainson R 2003. Flebotomíneos do Brasil, Fiocruz, Rio de Janeiro, $368 \mathrm{pp}$.

Rangel EF, Lainson R 2009. Proven and putative vectors of American cutaneous leishmaniasis in Brazil: aspects of their biology and vectorial competence. Mem Inst Oswaldo Cruz 104: 937-954. 
Ritchie MG 2007. Sexual selection and speciation. Annu Rev Ecol Evol Syst 38: 79-102.

Salomón OD, Quintana MG, Bezzi G, Morán ML, Betbeder E, Valdéz DV 2010. Lutzomyia migonei as putative vector of visceral leishmaniasis in La Banda, Argentina. Acta Trop 113: 84-87.

Souza NA, Andrade-Coelho CA, Vigoder FM, Ward RD, Peixoto AA 2008. Reproductive isolation between sympatric and allopatric Brazilian populations of Lutzomyia longipalpis s.l. (Diptera: Psychodidae). Mem Inst Oswaldo Cruz 103: 216-219.

Souza NA, Vigoder FM, Araki AS, Ward RD, Kyriacou CP, Peixoto AA 2004. Analysis of the copulatory courtship songs of Lutzomyia longipalpis in six populations from Brazil. J Med Entomol 41: 906-913.

Vigoder FM, Araki AS, Bauzer LG, Souza NA, Brazil RP, Peixoto AA 2010. Lovesongs and period gene polymorphisms indicate Lutzomyia cruzi (Mangabeira, 1938) as a sibling species of the
Lutzomyia longipalpis (Lutz and Neiva, 1912) complex. Infect Genet Evol 10: 734-739.

Ward RD, Phillips A, Burnet B, Marcondes CB 1988. The Lutzomyia longipalpis complex: reproduction and distribution. In Biosystematics of haematophagous insects, Oxford University Press, Oxford, p. 258-269.

Ward RD, Ribeiro AL, Ready PR, Murtagh A 1983. Reproductive isolation between different forms of Lutzomyia longipalpis (Lutz \& Neiva), (Diptera: Psychodidae), the vector of Leishmania Donovani Chagasi Cunha \& Chagas and its significance to kala-azar distribution in South America. Mem Inst Oswaldo Cruz 78: 269-280.

Young DG, Duncan MA 1994. Guide to the identification and geographic distribution of Lutzomyia sand flies in Mexico, the West Indies, Central and South America (Diptera: Psychodidae), Memories American Entomological Institute 54, Associated Publishers, Gainesville, 888 pp. 